\title{
Diagnosis and management of coronavirus disease-associated immune thrombocytopenia: a case series
}

\author{
Fatma Yılmaz Aydın ${ }^{[1]}$ and Vehbi Demircan ${ }^{[2]}$ \\ [1]. University of Dicle, School of Medicine, Department of Internal Medicine, Diyarbakır, Turkey. \\ [2]. University of Dicle, School of Medicine, Department of Hematology, Diyarbakır, Turkey.
}

\begin{abstract}
Data on health problems and fatal complications associated with coronavirus disease (COVID-19) have consistently been reported. Although immune thrombocytopenia has been associated with multiple viral infections, only few studies have shown its association with COVID-19. Here, we have reported a case series of two cases pertaining to patients diagnosed with COVID-19-associated immune thrombocytopenia, elaborating on the clinical course, management, and response to treatment.
\end{abstract}

Keywords: Immune thrombocytopenia. Thrombocytopenia. Coronavirus disease.

\section{INTRODUCTION}

Immune thrombocytopenia (ITP) is an uncommon disease with a platelet count of $<100 \times 10^{3} / \mathrm{uL}$, mostly with minor hemorrhages ${ }^{1}$. In this disease, isolated thrombocytopenia develops due to de novo or autoantibody-mediated destruction of platelets secondary to an underlying disorder. The causes of secondary ITP include autoimmune, lymphoproliferative, and collagen vascular diseases ${ }^{2}$. ITP is also associated with many infections, most of which are virus induced. Hepatitis $\mathrm{C}$ virus, human immunodeficiency virus, herpes viruses, cytomegalovirus, Epstein-Barr virus, parvovirus, measles, and rubella are common viral causes of ITP2 .

Coronavirus disease (COVID-19), caused by severe acute respiratory syndrome coronavirus 2 , which was first reported in December 2019, is a serious infection that has caused a global pandemic ${ }^{2}$. Data on various health problems and fatal complications caused by COVID-19 have been reported in the literature. Sepsis, in which the inflammatory response plays a role in pathogenesis, increases mortality ${ }^{3}$. Hematological problems including lymphopenia, thrombocytopenia, and disseminated intravascular coagulation (DIC) are common hematological conditions associated with COVID-19. Thrombocytopenia is a

\footnotetext{
Corresponding author: Fatma Yılmaz Aydın.

e-mail: fatmay83@hotmail.com

(i) https://orcid.org/0000-0002-8101-2497

Received 27 January 2021

Accepted 29 March 2021
}

serious risk factor for increased morbidity and mortality, especially in severe COVID-19 cases $^{4}$. However, the link between ITP and COVID-19 has rarely been reported in the literature ${ }^{4,5}$. Here, we have presented a case series of two cases pertaining to patients with COVID-19-associated ITP.

\section{CASE REPORT}

The first patient was a 42-year-old woman who presented with nasal bleeding and widespread petechiae and purpura on the skin. She had been diagnosed with epilepsy before 4 years. Three weeks before admission, the patient developed diarrhea and vomiting and was then diagnosed with COVID-19 on a polymerase chain reaction (PCR) test. She was administered hydroxychloroquine treatment for 5 days. Additionally, thorax computed tomography (CT) revealed bilateral ground-glass opacities. She was admitted to our hospital after multiple nosebleeds and petechiae purpura on the 21 st day. A laboratory blood test revealed a thrombocyte count of $2 \times 10^{3} / \mathrm{uL}$ (Table 1). Peripheral blood smear showed no schistocytes. After excluding DIC, microangiopathic diseases (such as thrombotic thrombocytopenia purpura and hemolytic uremic syndrome), other infections, drugs, and antiphospholipid antibodies that might have caused thrombocytopenia, the patient was diagnosed with COVID19-associated ITP. To control the severe thrombocytopenia, two units of platelet transfusion were administered. Treatment with prednisolone (1 mg/kg/day) and intravenous immunoglobulin (IVIG; $1 \mathrm{~g} / \mathrm{kg}$ ) was initiated. After 2 days of prednisolone and IVIG therapy, her platelet count increased to $41 \times 10^{3} / \mathrm{uL}$. Since the patient's platelet count was $453 \times 10^{3} / \mathrm{uL}$ and prednisolone treatment was continued for 1 week, the dose was slowly tapered and discontinued (Figure 1). 
TABLE 1: Characteristics of the two patients with COVID-19-associated ITP

\begin{tabular}{|c|c|c|c|}
\hline & Patient 1 & Patient 2 & Reference range \\
\hline Age (years) & 42 & 33 & \\
\hline Sex & Female & Female & \\
\hline Hemoglobin level (g/dL) & 9.7 & 13.5 & $12.9-14.2$ \\
\hline Platelet count $\left(\times 10^{3} / \mathrm{uL}\right)$ & 2 & 4 & $155-366$ \\
\hline Leucocyte count $\left(\times 10^{3} / \mathrm{uL}\right)$ & 10.2 & 7.19 & $3.7-10.1$ \\
\hline Lymphocyte count $\left(\times 10^{3} / \mathrm{uL}\right)$ & 0.58 & 0.67 & $1.09-2.99$ \\
\hline PT (s) & 12.5 & 12 & $10-14$ \\
\hline APTT (s) & 22.2 & 23.1 & $21-29$ \\
\hline D-dimer (mg/L) & 0.4 & 0.38 & $0.08-583$ \\
\hline Lupus anticoagulant & Negative & Negative & \\
\hline Anti-cardiolipin antibodies & Negative & Negative & \\
\hline Anti-nuclear antibodies & Positive & Negative & \\
\hline \multicolumn{4}{|l|}{ Virus serology } \\
\hline Human immunodeficiency virus & Negative & Negative & \\
\hline Hepatitis B and C & Negative & Negative & \\
\hline Epstein-Barr virus & Negative & Negative & \\
\hline Parvo $B 19$ virus & Negative & Negative & \\
\hline Cytomegalovirus virus & Negative & Negative & \\
\hline HSV virus & Negative & Negative & \\
\hline
\end{tabular}

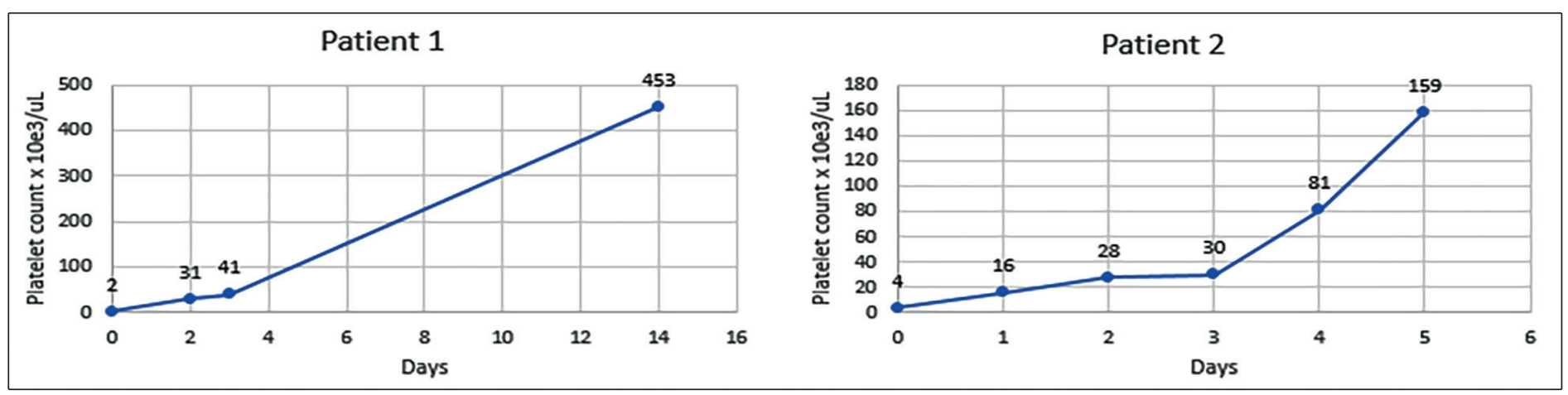

FIGURE 1: The course of platelet counts in two patients with COVID-19-associated ITP.

The second patient was a 33-year-old woman with a history of chronic hepatitis B infection. She presented with cough and fever. Subsequently, she was diagnosed with COVID-19 on a PCR test and was administered favipiravir treatment. The patient presented with petechiae, purpura, and severe vaginal bleeding on the four day after diagnosed with COVID-19. A laboratory test for complete blood count revealed a platelet count of $4 \times 10^{3} / \mathrm{uL}$ (Table 1), while the peripheral smear revealed normal findings, except for isolated thrombocytopenia. The patient underwent two units of apheresis platelet transfusion. Corticosteroid administration was considered, but not initiated due to insufficient and limited data at that time and the concern that it may worsen outcomes during the active replicative phase of COVID-19. Only two courses of IVIG $(1 \mathrm{~g} / \mathrm{kg})$ were administered. On the second day after initiation of
IVIG, her platelet count increased to $28 \times 10^{3} / \mathrm{uL}$. The patient was discharged after the platelet count was $159 \times 10^{3} / \mathrm{uL}$ on the 5 th day of the treatment (Figure 1). Based on the exclusion of other causes of ITP and the rapid improvement in platelet count with treatment, we diagnosed the patient with COVID-19-associated ITP.

\section{DISCUSSION}

The exact mechanism of ITP is not fully understood. Many researcher have suggested that viral infection triggers the disease and that pre-formed antibodies cross-react with platelet surface integrins such as glycoprotein Ib-IX-V or glycoprotein IIb/IIIa ${ }^{6,7}$. Although ITP has been associated with many viral infections ${ }^{8}$, there are limited data on its association with COVID-19. The diagnosis of COVID-19-associated ITP may be difficult due to several other 
potential causes, such as coagulation activation by COVID-19, leading to DIC and subsequent thrombocytopenia. Zulfiqar et al. have reported a case of COVID-19-associated ITP, which developed during the active phase of the infection ${ }^{5}$. Contrastingly, in a case series of three cases, Bomhof et al. found that ITP can develop not only during active infection but also after resolution of COVID-19 symptoms ${ }^{4}$. Thrombocytopenia developed in one of our patients approximately 2 weeks after recovery of COVID-19 symptoms, while the other patient developed ITP in the severe symptomatic period. Therefore, ITP can occur not only during active infection but also after many weeks of alleviation of COVID-19 symptoms.

The purpose of ITP therapy is to prevent severe bleeding by providing adequate platelet. ITP treatments include glucocorticoids, IVIG, or thrombopoietin receptor agonists ${ }^{9}$. IVIG is usually preferred in cases where a rapid increase in platelet count is required. Since IVIG inhibits the phagocytosis of macrophages, treatment with IVIG may be successful in the early stages of COVID- $19^{8}$. We preferred IVIG treatment in our patients primarily because it arrested severe thrombocytopenia and active bleeding. Glucocorticoids, another treatment option, are the primary management modality for ITP'. In our first patient, we used prednisolone treatment for 2 weeks in addition to IVIG treatment since thrombocytopenia developed after COVID-19 symptoms improved and the PCR test revealed negative results. We did not prefer thrombopoietin receptor agonists in our patients due to the high risk of thromboembolism in patients with severe COVID-19. Previously, corticosteroid administration was not recommended by the World Health Organization (WHO) for the treatment COVID- $19^{10}$. Historically, the use of corticosteroids during the severe acute respiratory syndrome and Middle East respiratory syndrome epidemics has been known to suppress the immune response ${ }^{11}$. Since then, evidence supporting benefits of corticosteroid treatment for some patients with COVID-19 has been increasing ${ }^{12}$.

Early diagnosis and treatment of this complication of COVID-19 can lead to better outcomes. Clinicians should adopt an individualized approach to evaluate the risks and benefits when initiating ITP treatments.

\section{ACKNOWLEDGMENTS}

The authors would like to thank everyone who contributed to the completion of this study.

\section{AUTHORS' CONTRIBUTION}

FYA: Conception and design of the study, Acquisition of data; Conception and design of the study, Analysis and interpretation of data, Final approval of the version to be submitted; Conception and design of the study, Acquisition of data, Drafting the article, Final approval of the version to be submitted; Conception and design of the study, Analysis and interpretation of data; VD: Conception and design of the study, Acquisition of data; Conception and design of the study, Analysis and interpretation of data.

\section{CONFLICT OF INTEREST}

The authors declare that there is no conflict of interest.

\section{FINANCIAL SUPPORT}

No financial support.

\section{ORCID}

Fatma Y1lmaz Aydın: 0000-0002-8101-2497

Vehbi Demircan: 0000-0002-0378-8687

\section{REFERENCES}

1. Rodeghiero F, Stasi R, Gernsheimer T, Michel M, Provan D, Arnold DM, et al. Standardization of terminology, definitions and outcome criteria in immune thrombocytopenic purpura of adults and children: report from an international working group. Blood. 2009;113(11):2386-93.

2. Liebman HA. Viral-associated immune thrombocytopenic purpura Hematology Am Soc Hematol Educ Program. 2008;212-8.

3. Aydın E, Yıldırım Y, Yılmaz Aydın F, Bahadır MV, Kaplan I, Kadiroğlu B, et al. Evaluation of the effect of intraperitoneal etanercept administration on oxidative stress and inflammation indicators in the kidney and blood of experimental sepsis-induced rats. Rev Soc Bras Med Trop. 2020;53:e20200016.

4. Bomhof G, Mutsaers PGNJ, Leebeek FWG, Boekhorst PAW, Hofland J, Croles FN, Jansen AJG. COVID-19-associated immune thrombocytopenia. Br J Haematol. 2020;190(2):e61-e64.

5. Zulfiqar AA, Lorenzo-Villalba N, Hassler P, Andrès E. Immune thrombocytopenic purpura in a patient with Covid-19. N Engl J Med. 2020;382(18):e43.

6. Taub JW, Warrier I, Holtkamp C, Beardsley DS, Lusher JM. Characterization of autoantibodies against the platelet glycoprotein antigens IIb/IIIa in childhood idiopathic thrombocytopenia purpura. Am J Hematol. 1995;48(2):104-7.

7. Mayer JLR, Beardsley DS. Varicella-associated thrombocytopenia: autoantibodies against platelet surface glycoprotein V. Pediatr Res. 1996;40(4):615-9.

8. Swinkels M, Rijkers M, Voorberg J, Vidarsson G, Leebeek FWG, Jansen AJG. Emerging concepts in immune thrombocytopenia. Front Immunol. 2018;9:880.

9. Cooper N, Ghanima W. Immune thrombocytopenia. N Engl J Med. 2019;381:945-55.

10. World Health Organization. Novel coronavirus (2019-nCoV) technical guidance: patient management. [May;2020].

11. Nasim S, Kumar S, Azim D, Ashraf Z, Azeem Q. Corticosteroid use for 2019-nCoV infection: A double-edged sword. Infect Control Hosp Epidemiol. 2020;41(10):1244-5.

12. Horby P, Lim SW, Emberson JR, Mafham M, Bell JL, Linsell L, et al. RECOVERY Collaborative Group. Dexamethasone in Hospitalized Patients with Covid-19. N Engl J Med. 2021;384(8):693-704. 